\title{
Investigation of the possible correlation between Idiopathic Parkinson's disease and Diabetes mellitus in Egyptian elderly patients (A pilot study)
}

\author{
Afnan Elgnainy ${ }^{1}$ \\ ${ }^{1}$ Misr University for Science and Technology
}

September 22, 2020

\author{
Keywords \\ Diabetes Mellitus; Parkinson's disease; Glucose kinetics
}

Registration numbers

clinicaltrials.gov number NCT03685357 